\section{Eltrombopag monotherapy can improve hematopoiesis in patients with low to intermediate risk-1 myelodysplastic syndrome}

\begin{abstract}
Alana Vicente,$^{1^{*}}$ Bhavisha A. Patel,${ }^{1 *}$ Fernanda Gutierrez-Rodrigues, ${ }^{1}$
Emma M. Groarke, ${ }^{1}$ Valentina Giudice, ${ }^{1}$ Jennifer Lotter, ${ }^{1}$ Xingmin Feng, ${ }^{1}$

Sachiko Kajigaya, ${ }^{1}$ Barbara Weinstein, ${ }^{1}$ Evette Barranta, ${ }^{1}$ Matthew J. OInes, ${ }^{1}$

Ankur R. Parikh, ${ }^{1}$ Maher Albitar, ${ }^{2}$ Colin 0. Wu, ${ }^{3}$ Ruba Shalhoub, ${ }^{3}$ Katherine R.

Calvo, ${ }^{4}$ Danielle M. Townsley, ${ }^{1}$ Phillip Scheinberg, ${ }^{5}$ Cynthia E. Dunbar, ${ }^{1}$ Neal S. Young ${ }^{1}$ and Thomas Winkler ${ }^{1}$
\end{abstract}

\begin{abstract}
${ }^{1}$ Hematology Branch, National Heart, Lung, and Blood Institute (NHLBI), National Institutes of Health (NIH), Bethesda, MD, USA; ${ }^{2}$ Genomics Testing Cooperative Laboratories, Irvine, CA, USA; ${ }^{3}$ Office of Biostatistics Research, National Institutes of Health (NIH), Bethesda, MD, USA; ${ }^{4}$ Hematology Section, Department of Laboratory Medicine, Clinical Center, National Institutes of Health (NIH), Bethesda, MD, USA and ${ }^{5}$ Hematology Department, Hospital A Beneficiencia Portuguesa, Sao Paulo, Brazil
\end{abstract}

${ }^{\star} A V$ and BAP contributed equally as co-first authors.

\section{ABSTRACT}

M yelodysplastic syndromes (MDS) are a group of clonal myeloid disorders characterized by low blood counts and a propensity to develop acute myeloid leukemia. The management of lowerrisk (LR) MDS with persistent cytopenias remains suboptimal. Eltrombopag, a thrombopoietin-receptor agonist, can improve platelet counts in LR-MDS and trilineage hematopoiesis in aplastic anemia. We conducted a phase II dose modification study to investigate the safety and efficacy of eltrombopag in LR-MDS. The eltrombopag dose was escalated from $50 \mathrm{mg} /$ day to a maximum of $150 \mathrm{mg}$ /day over a period of 16 weeks. The primary efficacy endpoint was hematologic response at 16-20 weeks. Eleven of 25 (44\%) patients responded; five and six patients had uni- or bi-lineage hematologic responses, respectively. The predictors of response were presence of a paroxysmal nocturnal hemoglobinuria clone, marrow hypocellularity, thrombocytopenia, and elevated plasma thrombopoietin levels at study entry. The safety profile was consistent with that found in previous eltrombopag studies in aplastic anemia; no patients discontinued the drug due to adverse events. Three patients developed reversible grade 3 liver toxicity and one patient had increased reticulin fibrosis. Ten patients discontinued eltrombopag after achieving a robust response (median time 16 months); four of them reinitiated eltrombopag because of declining blood counts, and all attained a second robust response. Six patients had disease progression not associated with expansion of mutated clones and no patient progressed to develop acute myeloid leukemia on study. In conclusion, eltrombopag was well-tolerated and effective in restoring hematopoiesis in some patients with low or intermediate-1 risk MDS. This study was registered at clinicaltrials.gov as \#NCT00932156.

\section{Introduction}

Myelodysplastic syndromes (MDS) are a heterogeneous group of clonal myeloid disorders characterized by ineffective hematopoiesis and cytopenias, with variable risks of progression to acute myelogenous leukemia (AML) ${ }^{1,2}$ The natural history of the disease is divergent between lower-risk (LR) and higher-risk MDS patients, evidenced by differences in clinical course, treatment efficacy, and overall survival. Higher-risk MDS appears close in pathophysiology to $\mathrm{AML}^{3}$ whereas LR-
Ferrata Storti Foundation

Haematologica 2020
Volume 105(12):2785-2794

\section{Correspondence:}

NEAL S. YOUNG

youngns@nhlbi.nih.gov

Received: March 24, 2020.

Accepted: May 21, 2020.

Pre-published: May 21, 2020.

doi:10.3324/haematol.2020.249995

(C)2020 NIH (National Institutes of Health) 
MDS is a more diverse group containing not only welldefined World Health Organization (WHO) classified categories but also subtypes that overlap with bone marrow failure syndromes, such as hypoplastic MDS (hypoMDS), MDS and paroxysmal nocturnal hemoglobinuria (PNH), and MDS evolved from aplastic anemia (AA). In these subtypes, T-cell-mediated suppression of hematopoiesis similar to that occurring in AA has been described. ${ }^{4-6}$

The prognosis of patients with MDS is determined using the International Prognostic Scoring System (IPSS) and the revised IPSS (IPSS-R), based on the degree of cytopenias, bone marrow blast percentage, and presence of specific cytogenetic abnormalities. ${ }^{7,8}$ Targeted nextgeneration sequencing has identified somatic variants in candidate genes associated with myeloid malignancies in more than $80 \%$ of MDS patients. ${ }^{9,10}$ Although the implications of these somatic variants in MDS have been extensively studied in the past years, most are not yet included in scoring systems.

MDS therapy is guided by IPSS risk stratification, with goals of treatment and tolerance of drug toxicity differing for higher risk-MDS and LR-MDS. In contrast to higher risk-MDS, supportive measures such as red blood cell transfusions, growth factors (erythropoiesis-stimulating agents and granulocyte colony-stimulating factor), and lenalidomide for patients with $\operatorname{del}(5 \mathrm{q})$ are common first options for LR-MDS. ${ }^{11-13}$ In addition, immunosuppressive treatments have demonstrated efficacy in LR-MDS, most notably in patients who are younger, HLA-DR15-positive, and have a more limited transfusion history. ${ }^{14,15}$ The treatment options for cytopenias in non-responders, especially for thrombocytopenia, are very limited, and such patients are often managed with long-term transfusion support. They remain at high risk of bleeding, developing infections, and having an overall poor quality of life.

Eltrombopag, a thrombopoietin-receptor agonist, was first used to treat thrombocytopenia in patients with idiopathic thrombocytopenic purpura, ${ }^{16}$ but has also been shown to improve hematologic response in patients with refractory severe AA and to increase overall and complete responses when combined with standard immunosuppression in treatment-naïve severe AA. ${ }^{17-20}$ In MDS, monotherapy with thrombopoietin agonists has only been tested in two studies, in which increased platelet counts were seen in nearly $50 \%$ of the patients. ${ }^{21,22} \mathrm{~A}$ randomized, double-blind study with romiplostim versus placebo for LR-MDS was stopped early due to an apparent increased risk of AML progression, which was not confirmed with long-term follow up. ${ }^{23,24}$ When eltrombopag was added to azacitidine to improve treatmentrelated thrombocytopenia in intermediate/high-risk $\mathrm{MDS}$, it resulted in worse platelet recovery and increased progression to AML. ${ }^{25}$

In this study, we investigated the safety and efficacy of eltrombopag monotherapy in LR-MDS and any cytopenia in a non-randomized phase II, investigator-initiated clinical trial.

\section{Methods}

\section{Patients and eligibility}

Subjects 18 years or older with LR-MDS were enrolled into this phase II, dose modification study of oral eltrombopag between March, 2011 and July, 2017. The protocol was approved by the Institutional Review Board of the National Heart, Lung, and Blood Institute, and monitored by an independent Data Safety and Monitoring Board.

The initial version of the protocol only included patients with platelet counts $\leq 30 \times 10^{\circ} / \mathrm{L}$ or platelet-transfusion dependence. After accrual of the first five patients, the inclusion criteria were broadened to enroll patients with any cytopenia. The revised inclusion criteria were: hemoglobin $\leq 9.0 \mathrm{~g} / \mathrm{dL}$ or red blood cell transfusion-dependence (at least 4 units of red blood cells at 8 weeks prior to enrollment); platelet counts $\leq 30 \times 10^{9} / \mathrm{L}$ or platelet transfusion-dependence; or absolute neutrophil count (ANC) $\leq 0.5 \times 10^{9} / \mathrm{L}$. Patients with refractory anemia with excess blasts, AML, treatment-related MDS, or chronic myelomonocytic leukemia were excluded.

\section{Treatment plan and study endpoints}

Patients received eltrombopag for 16-20 weeks. Eltrombopag was initiated at a dose of $50 \mathrm{mg}$ daily and the dose was increased to a maximum of $150 \mathrm{mg}$, unless toxicity-related stopping rules were met, dose reduction laboratory values occurred (Online Supplementary Table S1A,B), or hematologic response was achieved (Figure 1A). The primary safety endpoint was assessed using the National Cancer Institute's Common Terminology Criteria for Adverse Events version 4.0 (CTCAE $\mathrm{v} 4.0)$.

The primary efficacy endpoint was hematologic response at 16 weeks, defined as either: (i) a platelet count increase of $\geq 20 \times 10^{9} / \mathrm{L}$ above the baseline or stable platelet counts with transfusion-independence for $\geq 8$ weeks; (ii) a hemoglobin increase of $\geq 1.5 \mathrm{~g} / \mathrm{dL}$ or a reduction in red blood cell transfusion requirements by at least $50 \%$ over the preceding 8 weeks; (iii) $\geq 100 \%$ increase in ANC for those with a pretreatment ANC of $<0.5 \times 10^{9} / \mathrm{L}$ or an absolute increase $>0.5 \times 10^{9} / \mathrm{L}$. If patients had a clinical response in any lineage at 16 weeks but did not yet meet full primary endpoint criteria, eltrombopag was continued for another 4 weeks and response was assessed at 20 weeks.

Responding patients could receive eltrombopag on the extension arm until they met the criteria for a robust response (platelet count $>50 \times 10^{9} / \mathrm{L}$, hemoglobin $>10 \mathrm{~g} / \mathrm{dL}$, and ANC $>1.0 \times 10^{9} / \mathrm{L}$ ), at which time eltrombopag was discontinued. Eltrombopag was restarted in patients with blood counts falling below platelets $<30 \times 10^{9} / \mathrm{L}$, hemoglobin $<9 \mathrm{~g} / \mathrm{dL}$, or ANC $<0.5 \times 10^{9} / \mathrm{L}$.

Secondary endpoints were progression to higher-risk MDS, changes in serum thrombopoietin levels measured at the primary endpoint by magnetic multiplex assays (Luminex), ${ }^{26}$ eltrombopag discontinuation due to the achievement of a robust response, or grade 2 or higher bleeding events. International Working Group (IWG) criteria were used to determine the cytogenetic response and progression of disease. ${ }^{27}$

We screened all patients at baseline, at the primary endpoint, and at the time of disease progression for somatic variants in 63 candidate genes associated with myeloid malignancies using a targeted next-generation sequencing panel (Online Supplementary Table S2). ${ }^{28}$

\section{Statistics}

In this intention-to-treat study, summary statistics were used for patients' demographics and laboratory measurements. Covariate effects on the response rates and the distributions of survival time were evaluated using univariable logistic regression and Cox proportional hazard models, respectively. Further details on methods can be found in the Online Supplementary Methods. 


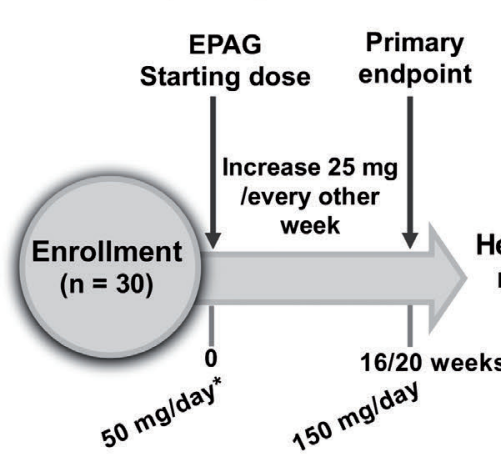

\section{Extended access}

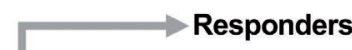

YES

Hematologic

response

NO

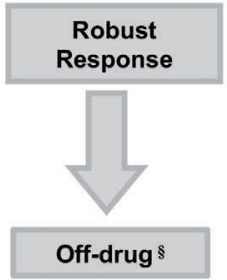

Off-study
B

\section{Primary endpoint ( $n=11)$}

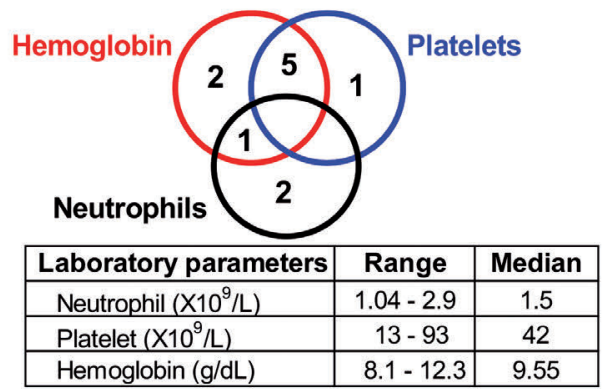

Best responses at follow-up $(n=14)$

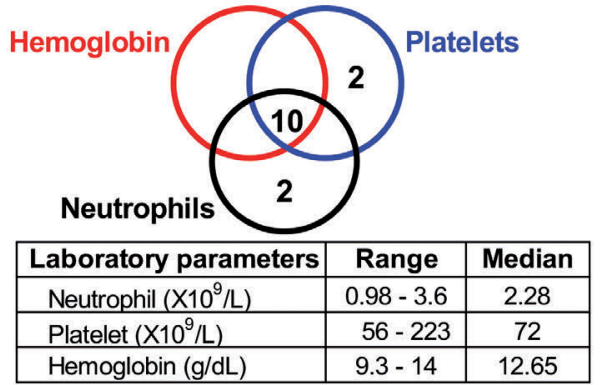

C

Hematologic improvement in all responders $(n=14)$
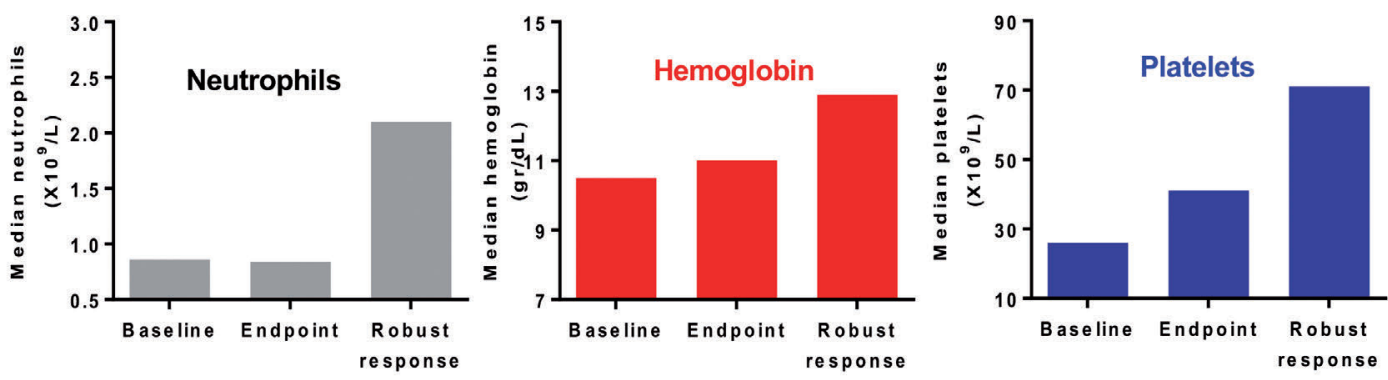

D

TPO levels at baseline and primary endpoint

\section{E TPO levels at baseline and primary endpoint}
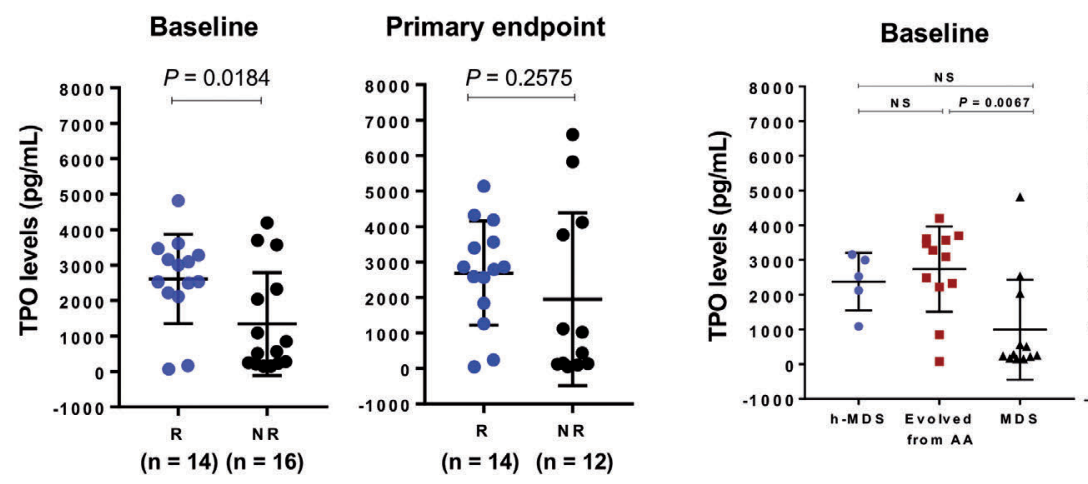

Primary endpoint

Figure 1. Study design and clinical outcomes of 30 patients with myelodysplastic syndrome. (A) Study flowchart. All patients enrolled on study received eltrombopag (EPAG) at a starting oral dose of $50 \mathrm{mg} /$ day, increased up to a maximum dose of $150 \mathrm{mg} /$ day for 16 weeks. Primary efficacy endpoint was assessed as hematologic improvement at 16-20 weeks. Non-responders were taken off study while responders continued EPAG in the extension phase until they achieved a protocol-defined robust response. If patients achieved a robust response, EPAG was discontinued and their blood counts and bone marrow values were monitored for 2 years. (B) Venn diagrams showing the number of patients with single lineage and multilineage responses to EPAG at the primary endpoints, and best responses in the extension phase. Laboratory parameters are also represented under the individual Venn diagrams. (C) Hematologic improvement of all responders, including the ten robust responders. The median neutrophil counts, hemoglobin concentration, and platelet levels are shown in the figure at the indicated time-points. (D) Thrombopoietin (TPO) levels of responders and non-responder at baseline and at the primary endpoint of the study. (E) TPO levels measured in patients with hypoplastic myelodysplastic syndrome (h-MDS), with myelodysplastic syndrome evolved from aplastic anemia (evolved from AA), and with normo- and hypercellular myelodysplastic syndrome (MDS) at study entry. NS: not statistically significant. 


\section{Results}

\section{Patients' characteristics and disposition}

A total of 30 patients were enrolled in the study and received eltrombopag. The first five subjects enrolled (UPN-1 to UPN-5) were entered when eligibility criteria included only thrombocytopenia. They were not included in the efficacy analysis set, as requested by the Institutional Review Board, but were included for secondary endpoint and sensitivity analyses.

In our cohort, $90 \%$ of patients were classified as IPSS intermediate-1 risk and as IPSS-R very low to intermediate risk (Table 1). Twenty-two patients $(73 \%)$ had either refractory cytopenia with multilineage dysplasia or refractory cytopenia with unilineage dysplasia. At enrollment, 11 patients had bicytopenia, ten had anemia (hemoglobin $<9.0 \mathrm{~g} / \mathrm{dL}$ ) or were red blood cell transfusion-dependent, and nine had thrombocytopenia (platelets $<30 \times 10^{\circ} / \mathrm{L}$ ) or were platelet-transfusion dependent (Table 2). Median blood counts for patients with anemia were hemoglobin $8.2 \mathrm{~g} / \mathrm{dL}$ (range, 7.1-11); with thrombocytopenia, platelets $11 \times 10^{9} / \mathrm{L}$ (range, 4-28), and neutropenia, $0.38 \times 10^{\%} / \mathrm{L}$. Twelve patients $(40 \%)$ had received at least one prior treatment other than supportive care and were considered to have relapsed/refractory disease. Prior therapies included lenalidomide, azacitidine, erythropoiesis-stimulating agents, and immunosuppressive treatments (Table 1). Four patients discontinued the study before the primary endpoint evaluation: UPN13 opted for supportive care, UPN-23 died from acute respiratory distress syndrome and mycobacterial infection, and UPN-19 and UPN-20 had worsening cytopenias with disease progression (described in more detail below and in Table 3).

\section{Safety}

In 25 of 30 (83\%) patients eltrombopag was escalated to the maximum dose $(150 \mathrm{mg}$ in all patients except the 3 patients of East or South-Asian origin). Of the five remaining patients, two had thrombocytosis at 75 $\mathrm{mg} /$ day requiring dose reduction (to $37.5 \mathrm{mg} /$ day), one patient had grade 3 elevated liver enzymes (alanine transaminase and aspartate transaminase $>5$ times the reference value) at a dose of $75 \mathrm{mg} /$ day which improved at a lower dose of $50 \mathrm{mg} / \mathrm{day}$, and two achieved platelet responses at lower doses ( $75 \mathrm{mg} /$ day and $125 \mathrm{mg}$ /day) so that dose escalation was halted per protocol. At the maximum dose of $150 \mathrm{mg}$, two patients experienced grade 3 reversible increases in liver transaminases, requiring dose interruption. After normalization of transaminases, eltrombopag was restarted at the lower dose level (125 $\mathrm{mg} /$ day) in both patients (UPN-4, UPN-18). The most frequent treatment-related adverse events were nausea and vomiting $(20 \%)$, skin lesions $(20 \%)$, headaches $(17 \%)$, and discoloration of the sclerae $(17 \%)$ (Online Supplementary Table S3).

There were no serious adverse events attributed to eltrombopag at the time of the data cut (Online Supplementary Table S4). One patient (UPN-24) with no response to treatment at the primary endpoint had increased reticulin fibrosis (from $1+$ to $3+$ ). Five patients $(17 \%)$ had grade 2 or higher bleeding adverse events at a median of 1 month (range, 0.34-4.5 months), which were not deemed to be related to eltrombopag, but to diseaseassociated thrombocytopenia. There were no eltrom- bopag-related deaths, thrombotic events, or progression to AML on study. One patient died due to acute respiratory distress syndrome unrelated to eltrombopag.

\section{Hematologic response}

Eleven of 25 patients (44\%) achieved a hematologic response at the primary endpoint; ten had been classified

Table 1. Baseline characteristics of the patients.

\begin{tabular}{|c|c|c|}
\hline Baseline characteristics & Cohort $(n=30)$ & Cohort $(n=25)$ \\
\hline $\begin{array}{l}\text { Age, years } \\
\text { Median (range) }\end{array}$ & $65(35-85)$ & $63(35-85)$ \\
\hline $\begin{array}{l}\text { Sex, n (\%) } \\
\text { Male } \\
\text { Female }\end{array}$ & $\begin{array}{l}21(70) \\
9(30)\end{array}$ & $\begin{array}{l}17(68) \\
8(32)\end{array}$ \\
\hline $\begin{array}{l}\text { Ethnicity, n (\%) } \\
\text { Asian } \\
\text { Black or African American } \\
\text { White } \\
\text { Other }\end{array}$ & $\begin{array}{l}3(10) \\
5(16.7) \\
21(70) \\
1(3.3)\end{array}$ & $\begin{array}{l}3(12) \\
4(16) \\
17(68) \\
1(4)\end{array}$ \\
\hline $\begin{array}{l}\text { WHO classification, n (\%) } \\
\text { RCUD } \\
\text { RCMD } \\
\text { MDS-U } \\
\text { RARS }\end{array}$ & $\begin{array}{l}11(36.7) \\
11(36.7) \\
6(20) \\
2(6.7)\end{array}$ & $\begin{array}{l}10(40) \\
9(36) \\
4(16) \\
2(8)\end{array}$ \\
\hline $\begin{array}{l}\text { IPSS risk, n (\%) } \\
\text { Low } \\
\text { Intermediate-1 } \\
\text { Intermediate-2 }\end{array}$ & $\begin{array}{r}2(6.7) \\
27(90) \\
1(3.3)\end{array}$ & $\begin{array}{l}2(8) \\
23(92) \\
0(0)\end{array}$ \\
\hline $\begin{array}{l}\text { IPSS-R risk, } \mathrm{n}(\%) \\
\text { Very low } \\
\text { Low } \\
\text { Intermediate } \\
\text { High } \\
\end{array}$ & $\begin{array}{l}1(3.3) \\
8(26.7) \\
18(60) \\
3(10)\end{array}$ & $\begin{array}{c}0(0) \\
8(32) \\
15(60) \\
2(8)\end{array}$ \\
\hline $\begin{array}{l}\text { IPSS cytogenetic risk classification*, } \\
\text { Good } \\
\text { Intermediate } \\
\text { Poor }\end{array}$ & $\begin{array}{c}16(53.3) \\
13(43.3) \\
1(3.3)\end{array}$ & $\begin{array}{c}14(56) \\
11(44) \\
0(0)\end{array}$ \\
\hline $\begin{array}{l}\text { Types of previous systemic therapy } \mathrm{f} \\
\text { Lenalidomide } \\
\text { Azacitidine } \\
\text { Erythropoietin-stimulating agents } \\
\text { Immunosuppressive therapy }\end{array}$ & $\begin{array}{l}\text { n (\%) } \\
\begin{aligned} 5(17) \\
6(20) \\
12(40) \\
1(3)\end{aligned}\end{array}$ & $\begin{array}{l}5(20) \\
3(12) \\
9(36) \\
0(0)\end{array}$ \\
\hline
\end{tabular}

Laboratory parameters

Neutrophil count, $\times 10^{9} / \mathrm{L}$

Median (range)

Platelet count, $\times 10^{\circ} / \mathrm{L}$

Median (range)

$0.995(0.26-3.77) \quad 1.06(0.26-3.77)$

Hemoglobin, g/dL

Median (range)

PNH clones, $n(\%)$

$\begin{array}{lll}\geq 1.0 \% & 11(36.7) & 10(40) \\ <1.0 \% & 19(63.3) & 15(60)\end{array}$

Thrombopoietin, $\mathrm{pg} / \mathrm{mL}$

Median (range)

$2119(71-4817) \quad 2080(71-4817)$

Transfusion dependency, $\mathrm{n}(\%)$

$\begin{array}{lll}\text { Platelets } & 16(53.3) & 11(44) \\ \text { Red cells } & 22(73.3) & 18(76)\end{array}$

$\overline{\text { All patients included in the study are shown in the left column }(\mathrm{n}=30) \text {, whereas the right column }}$ $(\mathrm{n}=25)$ shows the patients included in the primary endpoint analysis. WHO: World Health Organization; RCUD: refractory cytopenia with unilineage dysplasia; RCMD: refractory cytopenia with multilineage dysplasia; MDS-U: dysplasia; myelodysplastic syndrome-unclassifiable; RARS: refractory anemia with ringed sideroblasts; IPSS: International Prognostic Scoring System; IPSSR: Revised International Prognostic Scoring System; MDS: myelodysplastic syndrome: PNH: paroxysmal nocturnal hemoglobinuria. 
at baseline as IPSS intermediate-1 risk and one as low risk. The median time to response was 16 weeks (range, $16-20$ weeks). Both unilineage (5/11, 46\%) and bilineage $(6 / 11,55 \%)$ responses were seen at 16 weeks (Figure $1 \mathrm{~B}$ and Table 2). Eight of 11 responders were transfusion dependent for platelets and/or red blood cells before eltrombopag treatment and six of them became transfusion independent at 16 weeks. Three of 11 responders $(27 \%)$ showed normalization of a previously abnormal karyotype (trisomy 6, trisomy 15, and deletion 13q) at a median time of 20 months (range, 9-21 months) (Online Supplementary Table S5). Additionally, three of the first five patients enrolled (UPN-1, UPN-4, UPN-5) excluded from the efficacy analysis set achieved a platelet response and continued eltrombopag on the extension arm.

A total of 14 patients, including three of the first five enrolled patients excluded from the efficacy analysis set, continued to receive eltrombopag in the extension phase of the study at a median dose of $150 \mathrm{mg}$ /day (range, 37.5$150 \mathrm{mg}$ ). All 14 patients experienced further hematologic improvement (robust response or single lineage response) with longer treatment. At the primary endpoint, the median increase in hemoglobin levels was $1.4 \mathrm{~g} / \mathrm{dL}$ (range, 1.2-3.3), platelet numbers $14 \times 10^{9} / \mathrm{L}$ (range, $-12-$ 67 ) and neutrophil counts $0.71 \times 10^{9} / \mathrm{L}$ (range, $-0.2-2.52$ ) (Figure 1C). At best response, the median increase from baseline for hemoglobin was $4.45 \mathrm{~g} / \mathrm{dL}$ for platelets $53.5 \times 10^{9} / \mathrm{L}$ and the median increase in neutrophils was $1.14 \times 10^{9} / \mathrm{L}$. A robust response was achieved by ten of 14 patients with median drug administration of 16 months (range, 9-42 months) (Figure 1C), and eltrombopag was discontinued per protocol (Figure 2). Of these, four sus-

Table 2. Patients' characteristics and hematologic response to eltrombopag.

\begin{tabular}{|c|c|c|c|c|c|c|c|c|c|c|}
\hline \multirow[t]{2}{*}{ Age } & \multirow[t]{2}{*}{ Sex } & \multirow[t]{2}{*}{$\begin{array}{l}\text { WhO } \\
\text { sulbtype }\end{array}$} & \multirow[t]{2}{*}{$\begin{array}{l}\text { PNH } \\
\text { clone }\end{array}$} & \multirow[t]{2}{*}{ IPSS } & \multirow{2}{*}{$\begin{array}{c}\text { Bone } \\
\text { marrow } \\
\text { cellularity }\end{array}$} & $\begin{array}{l}\text { Cytopenias } \\
\text { at study entry }\end{array}$ & $\begin{array}{l}\text { Lineage responses } \\
\text { at primary endpoint }\end{array}$ & \multicolumn{3}{|c|}{ Robust cell counts } \\
\hline & & & & & & ANC Hb Pit & ANC Hb Pit & Months & ANC & Hb \\
\hline
\end{tabular}

\section{Responders}

\begin{tabular}{|c|c|c|c|c|c|c|c|c|c|c|c|c|c|c|c|c|}
\hline 1 & 65 & M & RCMD & $<1 \%$ & Int-1 & $35 \%$ & & $\mathrm{x}$ & $\mathrm{x}$ & & & $\mathrm{X}$ & 16 & $\mathrm{X}$ & $\mathrm{X}$ & $\mathrm{X}$ \\
\hline 4 & 79 & M & RCUD & $<1 \%$ & Int-2 & $5 \%$ & & & $\mathrm{X}$ & & & $\mathrm{X}$ & & & & \\
\hline 5 & 46 & $\mathrm{~F}$ & MDS-U & $4.60 \%$ & Int-1 & $<10 \%$ & & & $\mathrm{x}$ & & & $\mathrm{X}$ & 20 & $\mathrm{x}$ & $\mathrm{x}$ & $\mathrm{X}$ \\
\hline 6 & 53 & M & RCMD & $8.60 \%$ & Int-1 & $45 \%$ & & & $\mathrm{X}$ & & $\mathrm{X}$ & $\mathrm{X}$ & 16 & $\mathrm{X}$ & $\mathrm{X}$ & $\mathrm{X}$ \\
\hline 7 & 73 & $\mathrm{~F}$ & RCUD & $<1 \%$ & Int-1 & $15-20 \%$ & & & $\mathrm{X}$ & & $\mathrm{X}$ & $\mathrm{X}$ & 12 & $\mathrm{X}$ & $\mathrm{X}$ & $\mathrm{X}$ \\
\hline 11 & 35 & M & RCMD & $3.30 \%$ & Int-1 & $30 \%$ & & & $\mathrm{X}$ & & $\mathrm{x}$ & $\mathrm{X}$ & 12 & $\mathrm{X}$ & $\mathrm{X}$ & $\mathrm{X}$ \\
\hline 14 & 85 & M & RCUD & $<1 \%$ & Low & $25 \%$ & & & $\mathrm{X}$ & & & $\mathrm{X}$ & & & & \\
\hline 16 & 62 & $\mathrm{~F}$ & RCMD & $3 \%$ & Int-1 & $50 \%$ & & $\mathrm{X}$ & $\mathrm{X}$ & & $\mathrm{X}$ & $\mathrm{X}$ & 19 & $\mathrm{X}$ & $\mathrm{X}$ & $\mathrm{x}$ \\
\hline 17 & 54 & $\mathrm{~F}$ & RCUD & $<1 \%$ & Int-1 & $40 \%$ & & $\mathrm{X}$ & $\mathrm{X}$ & & $\mathrm{X}$ & $X$ & 9 & $\mathrm{X}$ & $\mathrm{X}$ & $\mathrm{X}$ \\
\hline 18 & 59 & $\mathrm{~F}$ & RCUD & $38.20 \%$ & Int-1 & $5 \%$ & & $\mathrm{X}$ & $\mathrm{X}$ & $\mathrm{X}$ & $\mathrm{X}$ & & 42 & $\mathrm{X}$ & $\mathrm{X}$ & $\mathrm{X}$ \\
\hline 25 & 72 & M & RCMD & $<1 \%$ & Int-1 & $80 \%$ & $\mathrm{x}$ & $\mathrm{X}$ & & $\mathrm{X}$ & & & & & & \\
\hline 26 & 36 & $\mathrm{~F}$ & MDS-U & $5 \%$ & Int-1 & $20 \%$ & & $\mathrm{X}$ & & & $\mathrm{X}$ & & 22 & $\mathrm{X}$ & $\mathrm{X}$ & $\mathrm{X}$ \\
\hline 27 & 47 & M & MDS-U & $5.80 \%$ & Int-1 & $30 \%$ & & $\mathrm{X}$ & $\mathrm{X}$ & & $\mathrm{x}$ & & 14 & $\mathrm{x}$ & $\mathrm{X}$ & $\mathrm{X}$ \\
\hline 30 & 47 & M & MDS-U & $4.80 \%$ & Int-1 & $5 \%$ & & $\mathrm{X}$ & $\mathrm{X}$ & $\mathrm{X}$ & & & & & & \\
\hline
\end{tabular}

\section{Non-responders}

\begin{tabular}{|c|c|c|c|c|c|c|c|c|}
\hline 2 & 76 & M & MDS-U & $<1 \%$ & Int-1 & $90 \%$ & & $\mathrm{X}$ \\
\hline 3 & 74 & M & RCMD & $<1 \%$ & Int-1 & $50 \%$ & & $\mathrm{X}$ \\
\hline 8 & 47 & M & MDS-U & $<1 \%$ & Int-1 & $5 \%$ & & $\mathrm{X}$ \\
\hline 9 & 67 & M & RCUD & $<1 \%$ & Int-1 & $5 \%$ & $\mathrm{X}$ & \\
\hline 10 & 76 & M & RCMD & $<1 \%$ & Int-1 & $50 \%$ & $\mathrm{X}$ & \\
\hline 12 & 76 & M & RARS & $<1 \%$ & Low & $90 \%$ & $\mathrm{X}$ & \\
\hline 13 & 54 & $\mathrm{~F}$ & RCUD & $1.50 \%$ & Int-1 & $40 \%$ & $\mathrm{X}$ & $\mathrm{x}$ \\
\hline 15 & 64 & $\mathrm{~F}$ & RCUD & $1.70 \%$ & Int-1 & $5 \%$ & $\mathrm{X}$ & $\mathrm{X}$ \\
\hline 19 & 55 & M & RCUD & $<1 \%$ & Int-1 & $50 \%$ & $\mathrm{X}$ & \\
\hline 20 & 63 & M & RCUD & $<1 \%$ & Int-1 & $60 \%$ & $\mathrm{X}$ & \\
\hline 21 & 68 & M & RCUD & $47.10 \%$ & Int-1 & $40 \%$ & $\mathrm{X}$ & $\mathrm{X}$ \\
\hline 22 & 72 & M & RARS & $<1 \%$ & Int-1 & $70 \%$ & $\mathrm{X}$ & \\
\hline 23 & 76 & M & RCMD & $<1 \%$ & Int-1 & $30 \%$ & $\mathrm{X}$ & \\
\hline 24 & 63 & $\mathrm{~F}$ & RCMD & $<1 \%$ & Int-1 & $40 \%$ & $\mathrm{X}$ & $\mathrm{X}$ \\
\hline 28 & 79 & M & RCMD & $<1 \%$ & Int-1 & $70 \%$ & $\mathrm{X}$ & \\
\hline 29 & 76 & M & RCMD & $<1 \%$ & Int-1 & $40 \%$ & $\mathrm{X}$ & \\
\hline
\end{tabular}

Cytopenia at baseline response at primary endpoint and time-point of a robust response are shown in this table for all 30 patients. UPN: unique patient number: WHO: World Health Organization; PNH: paroxysmal nocturnal hemoglobinuria; IPSS: International Prognostic Scoring System; ANC: absolute neutrophil count; Hb: hemoglobin; Plt: platelets; M: male; F: female; NR: non-response; RCMD: refractory cytopenia with multilineage dysplasia; RCUD: refractory cytopenia with unilineage dysplasia; MDS-U: myelodysplastic syndrome-unclassifiable; RARS: refractory anemia with ringed sideroblasts; int-1: intermediate 1 ; int-2: intermediate 2. 


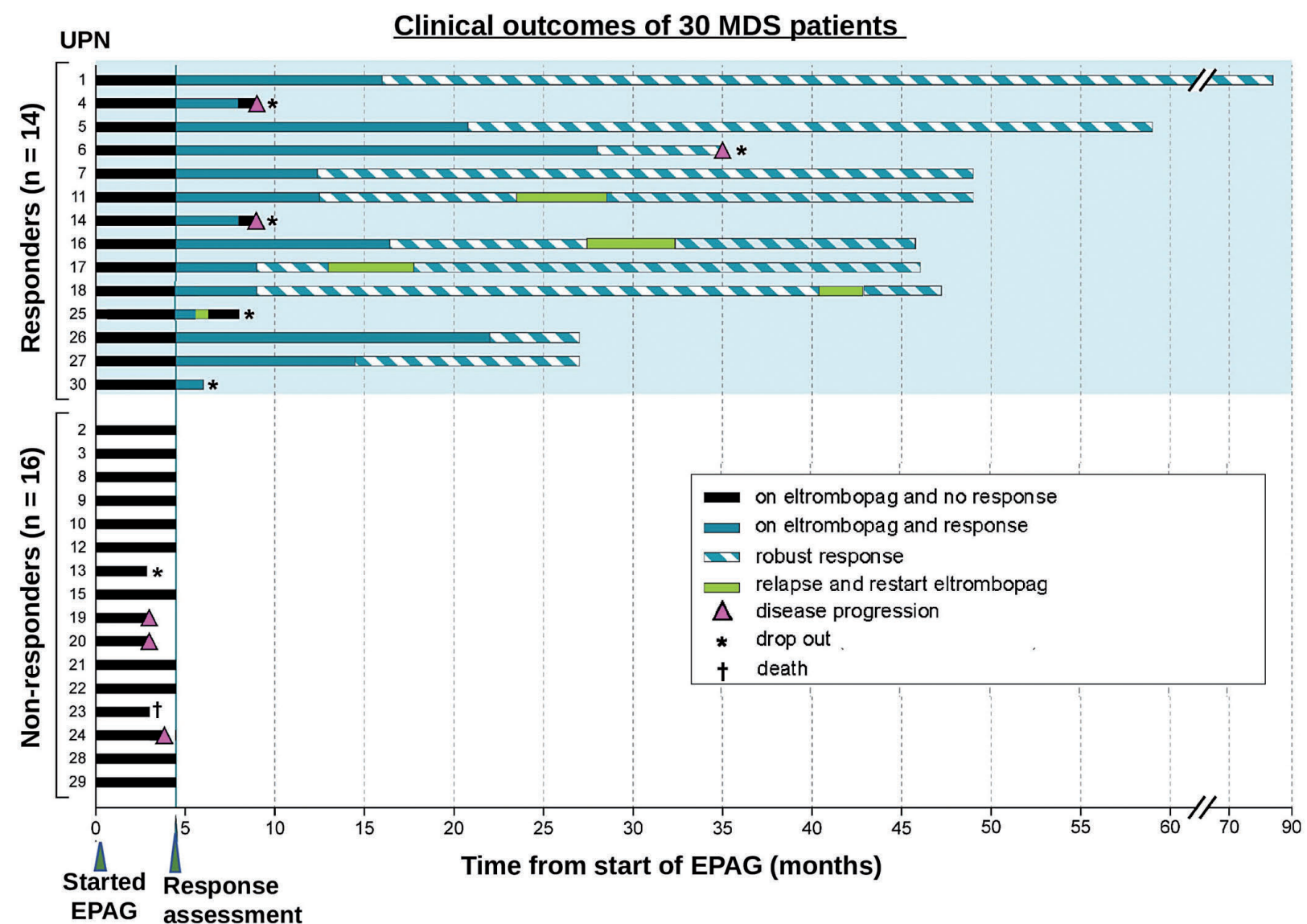

Figure 2. Clinical outcomes of the 30 patients with myelodysplastic syndrome enrolled in the study. Swimmer plot with the clinical outcomes of responders ( $=14$ ) and non-responders $(n=16)$. Bars represent the follow-up time for each patient. On the timeline, black bars represent the start of eltrombopag (EPAG) treatment until the primary outcome; blue bars represent the time for which patients continued EPAG on the extension arm; green bars represent the time that patients relapsed and restarted EPAG; pink triangles indicate the time of disease progression; dashed blue bars indicate when patients went off drug due to robust response. An asterisk indicates that the patient withdrew from the study, while a cross indicates that the patient died. MDS: myelodysplastic syndrome.

tained a hematologic response with a median follow up of 15 months off drug (range, 12-21 months). Declining counts were noted in four patients and eltrombopag was restarted at the last effective dose; all patients achieved a second robust response after a median of 12 months (range, 9-14 months) of additional eltrombopag treatment. At the time of data cut, eltrombopag was being tapered in all of these four patients. Of the remaining two robust responders, one developed a $\mathrm{PNH}$ clone and intravascular hemolysis, and another patient had disease progression.

Four of 14 patients who had achieved single lineage response at the primary endpoint sustained their response on the extension arm but discontinued treatment: one was lost to follow-up, one remained refractory to reinitiation of eltrombopag which was originally discontinued due to thrombocytosis, and two had progressive disease according to IWG criteria.

\section{Predictors of response}

On univariate analysis, the presence of more than 1\% glycosylphosphatidylinositol-deficient neutrophils $(P=0.036)$, thrombopoietin levels $\geq 2219 \mathrm{pg} / \mathrm{mL}(P=0.008)$, thrombocytopenia with or without other cytopenia $(P=0.015)$, and hypocellular marrow $(P=0.036)$ at baseline correlated with response to eltrombopag (Online
Supplementary Table S6A, B). Other baseline features such as age, absolute reticulocyte count, and ANC were not predictive. At baseline, median thrombopoietin plasma levels were significantly higher in patients who achieved response compared with levels in non-responders (median $2766 \mathrm{pg} / \mathrm{mL}$ vs. $562 \mathrm{pg} / \mathrm{mL}, P=0.018$ ) (Figure 1D). Among the responders, the two subjects with low thrombopoietin levels failed to achieve a robust response. At the primary endpoint, thrombopoietin levels remained elevated in responders compared to the levels in non-responders (median $2565 \mathrm{pg} / \mathrm{mL}$ vs. $1840 \mathrm{pg} / \mathrm{mL}$ ). High thrombopoietin levels were also associated with better survival according to Cox regression analysis (hazard ratio $<1$; $P=0.024$ ) (Online Supplementary Table S6C). We also compared thrombopoietin levels among MDS patients whose disease evolved from AA, who had hypo-MDS at diagnosis, or who had hyper/normocellular MDS. Hypo-MDS was defined as bone marrow cellularity $<30 \%$ in patients younger than 70 years or $<20 \%$ in those older than 70 years. Thrombopoietin levels in patients whose MDS evolved from AA were significantly higher than those in patients with de novo MDS at baseline and at the primary endpoint $(P=0.0067)$ (Figure 1E). The difference in thrombopoietin levels between hypo-MDS compared to hyper/normocellular MDS was not statistically significant $(P=0.12)$ (Figure 1E). Response rates in patients who had 
Table 3. Clinical characteristics of patients who progressed on study.

\begin{tabular}{|c|c|c|c|c|c|c|}
\hline & & Responders & & & Von-responders & \\
\hline & UPN-4 & UPN-6 & UPN-14 & UPN-19 & UPN-20** & UPN-24 \\
\hline Age, years & 79 & 53 & 85 & 55 & 63 & 63 \\
\hline Sex & M & M & M & M & M & $\mathrm{F}$ \\
\hline IPSS & Int-2 & Int-1 & Low & Int-1 & Int-1 & Int-1 \\
\hline $\begin{array}{l}\text { Baseline } \\
\text { Cytogenetics } \\
\text { Bone marrow blasts (\%) }\end{array}$ & $\begin{array}{c}45, \mathrm{XY},-7[20] \\
\quad<5 \%\end{array}$ & $\begin{array}{c}46, \mathrm{XY}[20] \\
<2 \%\end{array}$ & $\begin{array}{c}46, \mathrm{XY}[20] \\
<5 \%\end{array}$ & $\begin{array}{c}46, \mathrm{XY} \text {,del (5) } \\
\text { (q13q33) [20] } \\
<5 \%\end{array}$ & $\begin{array}{l}46, X Y, t(1 ; 9) \\
(p 34 ; q 22)[20] \\
<5 \%\end{array}$ & $\begin{array}{c}46, \mathrm{XX}[20] \\
<5 \%\end{array}$ \\
\hline $\begin{array}{l}\text { Baseline or best response } \\
\text { ANC }\left(\mathrm{x} 10^{9} / \mathrm{L}\right) \\
\text { Hemoglobin }(\mathrm{g} / \mathrm{dL}) \\
\text { Platelets }\left(\mathrm{x} 10^{9} / \mathrm{L}\right)\end{array}$ & $\begin{array}{l}0.71 \\
8.6 \\
70\end{array}$ & $\begin{array}{c}3.6 \\
12.9 \\
64\end{array}$ & $\begin{array}{c}4.9 \\
12.9 \\
93\end{array}$ & $\begin{array}{l}1.62 \\
8.9 \\
202\end{array}$ & $\begin{array}{l}1.64 \\
9.1 \\
93\end{array}$ & $\begin{array}{l}1.1 \\
11 \\
20\end{array}$ \\
\hline $\begin{array}{l}\text { Disease progression* } \\
\text { Cytogenetic }\end{array}$ & $45, \mathrm{XY},-7[20]$ & $47, X Y,+21[11] / 46, X Y[9]$ & NA & $\begin{array}{l}\text { 46,XY[3]/46,XY,del(5) } \\
\text { (q13q33) [9]/47,idem, } \\
\text { +21[5],46,idem, } \\
\text { I(21)(q10) [5] }\end{array}$ & $\begin{array}{l}46, X Y, t(1 ; 9) \\
(p 34 ; q 22)[20]\end{array}$ & $46, X X[20]$ \\
\hline $\begin{array}{l}\text { Bone marrow blasts }(\%) \\
\text { ANC }\left(x 10^{\%} / \mu \mathrm{L}\right) \\
\text { Hemoglobin }(\mathrm{g} / \mathrm{dL}) \\
\text { Platelets }\left(\mathrm{x} 10^{9} / \mu \mathrm{L}\right)\end{array}$ & $\begin{array}{l}8 \% \\
0.4 \\
9.9 \\
22\end{array}$ & $\begin{array}{c}6 \% \\
1.92 \\
13 \\
46\end{array}$ & $\begin{array}{c}\text { NA } \\
3.9 \\
13.1 \\
10\end{array}$ & $\begin{array}{c}<5 \% \\
1.13 \\
8.9 \\
66\end{array}$ & $\begin{array}{c}<5 \% \\
2.1 \\
9.1 \\
43\end{array}$ & $\begin{array}{c}<5 \% \\
0.82 \\
10 \\
7\end{array}$ \\
\hline Time on eltrombopag (months) & 9 & 28 & 9 & 3 & 3 & 4 \\
\hline Time to progression (months) & 9 & 35 & 9 & 3 & 3 & 4 \\
\hline Present status & Deceased & Alive & Deceased & Alive & Alive & Alive \\
\hline
\end{tabular}

*According to the modified 2006 International Working Group criteria; **UPN-20 was noted to have peripheral blasts at the time of progression; UPN: unique patient number; M: male; F: female; IPSS: International Prognostic Scoring System; Int-1: intermediate 1; Int-2: intermediate; NA: not available; BM: bone marrow; ANC: absolute neutrophil count; $\mathrm{Hb}$ : hemoglobin.

been previously treated were $20 \%$ after lenalidomide, $33 \%$ after hypomethylating agents and $50 \%$ after erythropoiesis-stimulating agents (Online Supplementary Table S7).

\section{Disease progression}

Of all 30 patients enrolled, six (20\%) had disease progression with a median time to progression of 6.5 months (range, 3-35 months). Three responding patients progressed during the extension phase of the study with a median time to progression of 9 months (range, 9-35 months) (Table 3).

UPN-4, who presented with IPSS intermediate- 2 and deletion $7 \mathrm{q}$ at baseline, was deemed a responder at the primary endpoint but platelet counts later declined and myeloblasts increased from $<5 \%$ to $8 \%$ after 9 months of eltrombopag treatment. The patient died from infectious complications after discontinuation of eltrombopag while receiving supportive care. Platelets and ANC declined in another responding patient 7 months after discontinuation of eltrombopag because of the patient's robust response; evaluation of the bone marrow revealed an increase in blasts and acquisition of trisomy 21 . This patient underwent successful allogeneic stem cell transplant. In UPN-14, platelet counts fell more than $50 \%$ at 9 months on eltrombopag, and the patient died from bleeding 1 month after stopping the drug; we were unable to evaluate his bone marrow at the time of disease progression.

Among the non-responders, three patients had disease progression at the time of the primary endpoint evaluation based on a decline in platelet counts by more than $50 \%$ when compared to the laboratory values at study entry (Table 3). None of these patients had increased blast percentage. In addition, UPN-19 had acquired a complex karyotype at the primary endpoint assessment. UPN-19 and UPN-20 underwent allogeneic stem cell transplantation and are alive. UPN-24 remained dependent on platelet transfusions at the 6-month follow up after discontinuing eltrombopag.

Furthermore, two non-responding patients with an abnormal baseline karyotype developed additional chromosome abnormalities (monosomy 7 in UPN-2 and a complex karyotype in UPN-3) at 16 weeks but did not meet IWG criteria for disease progression. UPN-2 died from AML 5 years after acquiring monosomy 7 and UPN3 died of bleeding complications 9 months after going off study.

\section{Somatic variants in myeloid candidate genes}

At baseline, 23 of 29 patients (52\%) were identified with somatic variants in genes recurrently mutated in myeloid malignancies. The most commonly mutated genes were related to epigenetic regulators and splicing factors, such as ASXL1 (21\%), TET2 $(17 \%)$, and SF3B1 (14\%) (Figure 3A).

At the primary endpoint, variants were found in six responders and seven non-responders (13 of 24 patients; $54 \%$ ) (Figure 3A). Novel variants were identified in two responders (UPN-14 and UPN-4) and in three non-responders (UPN-2, UPN-9, and UPN-12). Moreover, somatic variants in DNMT3A, BCOR, SETBP1, and ASXL1 at baseline were no longer detected at the primary endpoint in three non-responders (UPN-24, UPN-8, and UPN-2) (Figure 3A).

We investigated whether eltrombopag promoted the expansion of clones identified at baseline. We found no difference in the allele frequencies of variants detected 
before and after eltrombopag, regardless of the patients' response $(P=0.85)$ (Figure $3 \mathrm{~B}, \mathrm{C})$. No particular gene was associated with either expansion or reduction in the size of clones. Among four patients who progressed on study, according to IWG criteria, and had samples available for longitudinal analysis, only two acquired novel clones at progression (UPN-6 and UPN-20) (Online Supplementary Figure S2). At progression, a novel ASXL1 clone was found in UPN-20. UPN-6 progressed with trisomy 217 months after eltrombopag had been halted because of robust response, with concomitant expansion of the
ASXL1 clone (variant allele frequency of 24\%-39\%) and acquisition of a $R U N X 1$ variant (variant allele frequency of $54 \%$ ). Other clones identified at baseline remained stable or were no longer detected after progression (Figure 3A and Online Supplementary Figure S2).

\section{Discussion}

Our prospective, phase II study shows the efficacy of eltrombopag in inducing multilineage hematologic

A

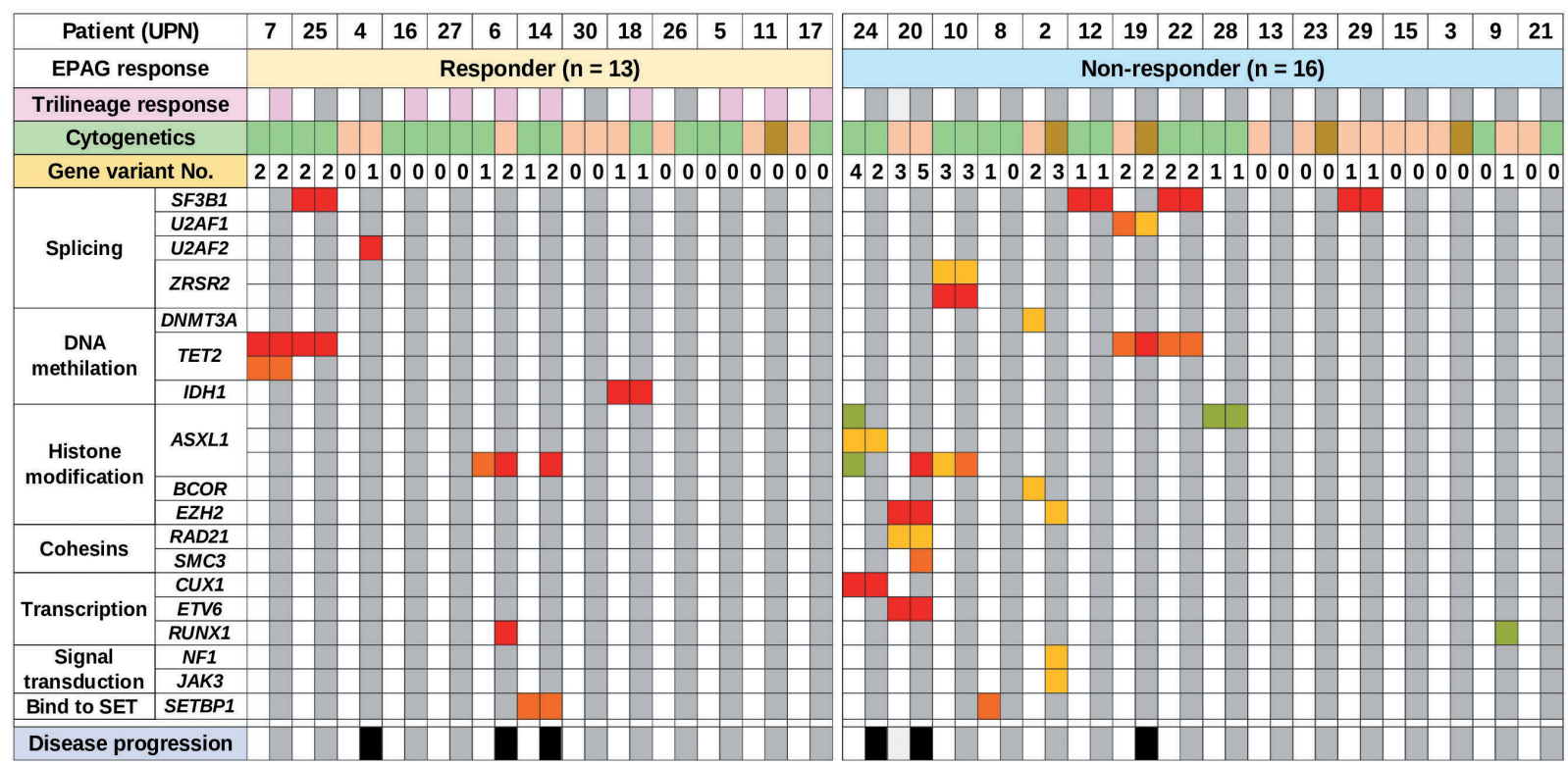

$\square$ Trilineage; $\square$ Normal cytogenetics; $\square$ Abnormal cytogenetics; $\square$ Different abnormal cytogenetics; Variant frequency (\%): $\square \geq 30, \square \geq 20$ to $<30, \square \geq 10$ to $<20, \square \geq 2.5$ to $<10$; Disease progressed

B

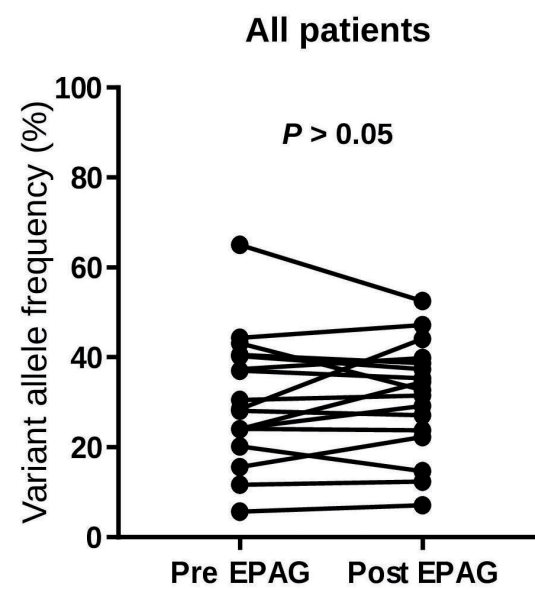

C

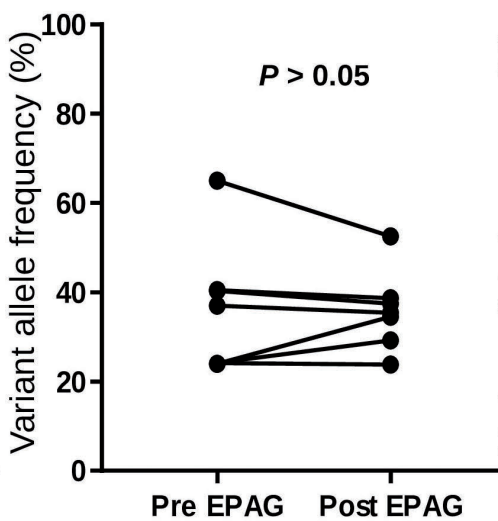

Non-Responders

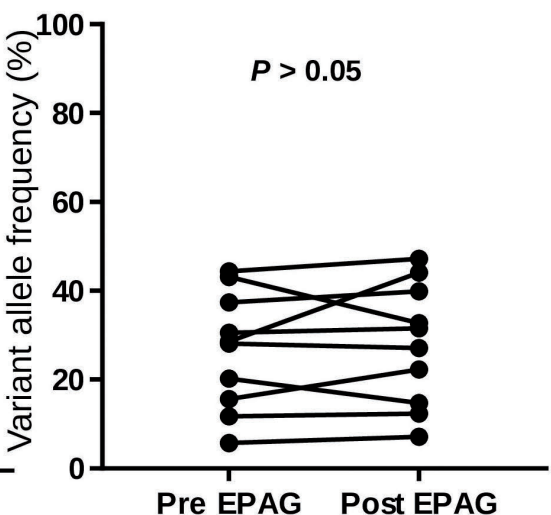

Figure 3. Clonal dynamics of variants identified in myeloid genes during the study. We screened 29 patients at baseline and 24 patients with samples available at the primary endpoint for somatic variants in genes recurrently mutated in myeloid neoplasia by targeted next-generation sequencing (Online Supplementary Table S2). (A) Summary of somatic variants identified in enrolled patients at baseline and at the primary endpoint. For each patient, white and gray columns represent the baseline and primary endpoints (16 or 20 weeks) on eltrombopag (EPAG), respectively. For each time-point, the trilineage responses, normal or abnormal cytogenetics, the frequency of somatic variants, and disease progression status are represented by different colors specified under the panel. Patients were grouped into responders and non-responders after EPAG treatment and then sorted by variant numbers (highest to lowest). The variant names, the number of variants, and gene functions are also indicated in the panel for each patient. Of note, other than a single nonsense variant, all the ASXL1 mutations were frameshift. Also, both patients with mutated SF3B1 had the K700E variant. (B) Variant allele frequencies of the somatic variants identified at baseline (pre-EPAG) and at the primary endpoint (post-EPAG) in the entire cohort. (C) Variant allele frequencies of somatic clones identified pre- and post-EPAG according to the patients' response. 
responses in about half of LR-MDS patients. Moreover, peripheral blood cell counts continued to improve with longer treatment duration and were sustained in some patients after discontinuation of eltrombopag. Our results confirm and extend observations of previous studies with thrombopoietin agonists, eltrombopag and romiplostim, which demonstrated platelet responses and reduction of thrombocytopenia-related adverse events in patients with LR-MDS and low platelet counts..$^{21,22}$ The quality of the hematologic response, with one-third of patients achieving a robust resposne, is encouraging, particularly considering that $40 \%(12 / 30)$ of patients had failed more than two lines of prior therapies. Furthermore, counts remained stable even after eltrombopag was discontinued and all patients who restarted eltrombopag achieved a second response. Remarkably, 20\% (3/14) of the responding patients in our cohort achieved a major cytogenetic response according to IWG 2006 criteria. Although this response was noted in small clones with abnormal karyotypes (Online Supplementary Table S5), these findings may indicate that eltrombopag preferentially stimulates normal hematopoietic stem and progenitor cells.

The toxicity profile in this study is comparable to that in previous studies in bone marrow failure, ${ }^{17-20}$ with only a few instances of temporary dose interruptions because of transient elevations of liver transaminases. Increased reticulin fibrosis (grade 1 to grade 3) was noted in one patient with disease progression, which could not be clearly attributed to either the study drug eltrombopag or underlying disease.

Baseline characteristics of a PNH clone, elevated thrombopoietin levels, thrombocytopenia with or without another cytopenia, and low marrow cellularity correlated with response to eltrombopag are novel findings in our study. Patients with a previous history of AA or hypoMDS at diagnosis may benefit from eltrombopag treatment more than do patients with more typical hyper/normocellular MDS (Online Supplementary Table S6B). The efficacy of immunosuppressive treatments and eltrombopag in AA and a group of LR-MDS patients suggests the existence of similar pathological mechanisms in these syndromes. ${ }^{14,29}$ Eltrombopag has been reported to modulate $\mathrm{T}$ regulatory cells, restore $\mathrm{Fc}-\gamma$ receptor balance in phagocytes, and to mobilize intracellular iron, ${ }^{30-32}$ but the exact mechanism of any interaction between eltrombopag and the immune system needs further investigation.

Despite the benefit of eltrombopag in improving cytopenias in patients with LR-MDS, one major concern regarding the use of thrombopoietin mimetics in myeloid malignancies is the expansion and stimulation of malignant clones. We found no correlation between patients' somatic gene mutation profile and hematologic response or progression of disease in our study. Our cohort included a large number of patients with hypo-MDS at diagnosis and whose MDS evolved from AA, some with the other features of immune-mediated marrow failure (PNH clone, elevated thrombopoietin levels, marrow hypocellularity), but overall the somatic mutation profile was representative of MDS. Frequently mutated genes were ASXL1 (21\%), TET2 (17\%) and SF3B1 (14\%), a different profile from that typical of AA $B C O R, B C O R L 1, P I G A$, and DNMT3A). ${ }^{33}$

No patient progressed to AML on study. One patient developed AML after having been off study for 5 years, consistent with the natural history of MDS, and this event was most likely not due to the earlier brief course of eltrombopag. Similar to our results, eltrombopag monotherapy was also not associated by others with an increased progression to AML in LR-MDS patients, ${ }^{21}$ being reported only in high-risk patient populations (those with refractory anemia with excess blasts-1 and -2 with romiplostim treatment), with a higher dose of eltrombopag (300 mg/day), and combination therapy with azacytidine. ${ }^{25,34}$ Six patients progressed on study $(20 \%)$, comprising three responders and three non-responders. The rate of progression observed in our study is similar to that in a previous eltrombopag trial $(12 \%){ }^{21}$ There was a difference in the timing and the type of progression between responders and non-responders; in non-responders the only criterion for progression before or at the time of the primary efficacy assessment was a decline in platelets, whereas responders had both cytopenia and an increased percentage of blasts during the extension phase. While eltrombopag at $150 \mathrm{mg} /$ day did not appear to result in progression to AML in LR-MDS patients, caution is indicated in the treatment of individual patients and further clinical studies are warranted. Our conclusions do not apply to eltrombopag in patients with high-risk IPSS scores or high-risk cytogenetics irrespective of IPSS. Until further data are available, close monitoring of peripheral blood counts, and frequent bone marrow and cytogenetic evaluations should be performed while patients are on eltrombopag.

The appearance of transient cytogenetically abnormal clones was observed in patients during the extension arm, a phenomenon that has also been reported in treatmentnaive AA patients after immunosuppressive treatment alone and with eltrombopag monotherapy for refractory $\mathrm{AA} .^{35}$ In MDS, some transient clones seem to be associated with better outcomes and may reflect momentary episodes of genetic instability, not of long-term clinical significance. ${ }^{36}$ In addition, no clonal expansion was noted after treatment with eltrombopag in either responders or non-responders in our trial.

In conclusion, our results indicate that eltrombopag as monotherapy is well tolerated and can be effective treatment for patients with low to intermediate-1 risk MDS. Our study not only confirmed the previously reported platelet response ${ }^{27}$ but showed robust and durable trilineage responses. Hypocellular marrow, elevated thrombopoietin, and a PNH clone predicted response to eltrombopag treatment. The main limitations of the study are the small sample size and the unique patients' characteristics resulting from the referral pattern of our institution. Further larger, prospective and controlled studies are warranted to better define the role of eltrombopag in the treatment of LR-MDS.

\section{Acknowledgments}

The authors gratefully acknowledge NIH physicians, nurses and other patient care providers involved. We thank co-investigators Dr. Elaine Sloand, Dr. Zhijie Wu and Dr. Carrie Diamond for their participation and contributions; and the Flow Cytometry Core at NHLBI. This research was funded by the Intramural Research Program of the NHLBI. Eltrombopag was provided by GlaxoSmithKline (Collegeville, PA, USA) and subsequently by Novartis (East Hanover, NJ, USA). 


\section{References}

1. Goasguen JE, Bennett JM. Classification and morphologic features of the myelodysplastic syndromes. Semin Oncol. 1992;19(1):4-13.

2. Ganser A, Hoelzer D. Clinical course of myelodysplastic syndromes. Hematol Oncol Clin North Am. 1992;6(3):607-618.

3. Sekeres MA, Cutler C. How we treat higher-risk myelodysplastic syndromes. Blood. 2014;123(6):829-836.

4. Huang TC, Ko BS, Tang JL, et al. Comparison of hypoplastic myelodysplastic syndrome (MDS) with normo-/hypercellular MDS by International Prognostic Scoring System, cytogenetic and genetic studies. Leukemia. 2008;22(3):544-550.

5. Young NS. Aplastic anemia. N Engl J Med. 2018;379(17):1643-1656.

6. Kasahara S, Hara $\mathrm{T}$, Itoh $\mathrm{H}$, et al. Hypoplastic myelodysplastic syndromes can be distinguished from acquired aplastic anaemia by bone marrow stem cell expression of the tumour necrosis factor receptor. Br J Haematol. 2002;118(1):181-188.

7. Greenberg P, Cox C, LeBeau MM, et al. International scoring system for evaluating prognosis in myelodysplastic syndromes. Blood. 1997:89(6):2079-2088.

8. Greenberg PL, Tuechler H, Schanz J, et al. Revised international prognostic scoring system for myelodysplastic syndromes. Blood. 2012;120(12):2454-2465.

9. Bejar R. Clinical and genetic predictors of prognosis in myelodysplastic syndromes. Haematologica. 2014;99(6):956-964.

10. Nazha A, Seastone D, Radivoyevitch $T$, et al. Genomic patterns associated with hypoplastic compared to hyperplastic myelodysplastic syndromes. Haematologica. 2015;100(11):e434-437.

11. List A, Dewald G, Bennett J, et al. Lenalidomide in the myelodysplastic syndrome with chromosome $5 \mathrm{q}$ deletion. N Engl J Med. 2006;355(14):1456-1465

12. Almeida A, Fenaux P, List AF, Raza A, Platzbecker U, Santini V. Recent advances in the treatment of lower-risk non-del $(5 \mathrm{q})$ myelodysplastic syndromes (MDS). Leuk Res. 2017;52:50-57.

13. Fenaux P, Adès L. How we treat lower-risk myelodysplastic syndromes. Blood. 2013; 121(21):4280-4286.

14. Stahl M, DeVeaux M, de Witte T, et al. The use of immunosuppressive therapy in MDS: clinical outcomes and their predictors in a large international patient cohort. Blood Adv. 2018;2(14):1765-1772.

15. Molldrem JJ, Caples M, Mavroudis D, Plante M, Young NS, Barrett AJ. Antithymocyte globulin for patients with myelodysplastic syndrome. Br J Haematol. 1997;99(3):699. 705.

16. Bussel JB, Cheng G, Saleh MN, et al. Eltrombopag for the treatment of chronic idiopathic thrombocytopenic purpura. N Engl J Med. 2007;357(22):2237-2247.

17. Olnes MJ, Scheinberg P, Calvo KR, et al. Eltrombopag and improved hematopoiesis in refractory aplastic anemia. N Engl J Med. 2012;367(1):11-19.

18. Desmond R, Townsley DM, Dumitriu B, et al. Eltrombopag restores trilineage hematopoiesis in refractory severe aplastic anemia that can be sustained on discontinuation of drug. Blood. 2014;123(12):1818-1825.

19. Townsley DM, Scheinberg P, Winkler T, et al. Eltrombopag added to standard immunosuppression for aplastic anemia. N Engl J Med. 2017;376(16):1540-1550.

20. Winkler T, Fan X, Cooper J, et al. Treatment optimization and genomic outcomes in refractory severe aplastic anemia treated with eltrombopag. Blood. 2019;133(24): 2575-2585.

21. Oliva EN, Alati C, Santini V, et al. Eltrombopag versus placebo for low-risk myelodysplastic syndromes with thrombocytopenia (EQoL-MDS): phase 1 results of a single-blind, randomised, controlled, phase 2 superiority trial. Lancet Haematol. 2017;4(3):e127-e136.

22. Kantarjian H, Fenaux P, Sekeres MA, et al. Safety and efficacy of romiplostim in patients with lower-risk myelodysplastic syndrome and thrombocytopenia. J Clin Oncol. 2010;28(3):437-444

23. Giagounidis A, Mufti GJ, Fenaux P, et al. Results of a randomized, double-blind study of romiplostim versus placebo in patients with low/intermediate-1-risk myelodysplastic syndrome and thrombocytopenia. Cancer. 2014;120(12):1838-1846.

24. Kantarjian HM, Fenaux P, Sekeres MA, et al. Long-term follow-up for up to 5 years on the risk of leukaemic progression in thrombocytopenic patients with lower-risk myelodysplastic syndromes treated with romiplostim or placebo in a randomised double-blind trial. Lancet Haematol. 2018;5(3):e117-e126.

25. Dickinson M, Cherif H, Fenaux P, et al. Azacitidine with or without eltrombopag for first-line treatment of intermediate- or high-risk MDS with thrombocytopenia. Blood. 2018;132(25):2629-2638.

26. Feng $\mathrm{X}$, Scheinberg $\mathrm{P}, \mathrm{Wu} \mathrm{CO}$, et al Cytokine signature profiles in acquired aplastic anemia and myelodysplastic syndromes. Haematologica. 2011;96(4):602-606.

27. Cheson BD, Greenberg PL, Bennett JM, et al. Clinical application and proposal for modification of the International Working Group (IWG) response criteria in myelodysplasia. Blood. 2006;108(2):419-425.

28. Albitar A, Townsley D, Ma W, et al Prevalence of somatic mutations in patients with aplastic anemia using peripheral blood cfDNA as compared with BM. Leukemia. 2018;32(1):227-229.

29. Saunthararajah Y, Nakamura R, Wesley R, Wang OJ, Barrett AJ. A simple method to predict response to immunosuppressive therapy in patients with myelodysplastic syndrome. Blood. 2003;102(8):3025-3027.

30. Liu XG, Liu S, Feng $Q$, et al. Thrombopoietin receptor agonists shift the balance of Fcy receptors toward inhibitory receptor IIb on monocytes in ITP. Blood. 2016;128(6):852-861.

31. Schifferli A, Nimmerjahn F, Kühne T. Immunomodulation in primary immune thrombocytopenia: a possible role of the Fc fragment of romiplostim? Front Immunol. 2019;10:1196

32. Roth M, Will B, Simkin G, et al. Eltrombopag inhibits the proliferation of leukemia cells via reduction of intracellular iron and induction of differentiation. Blood. 2012;120(2):386-394.

33. Yoshizato T, Dumitriu B, Hosokawa K, et al. Somatic mutations and clonal hematopoiesis in aplastic anemia. N Engl J Med. 2015;373 (1):35-47.

34. Kantarjian HM, Giles FJ, Greenberg PL, et al Phase 2 study of romiplostim in patients with low- or intermediate-risk myelodysplastic syndrome receiving azacitidine therapy. Blood. 2010;116(17):3163-3170

35. Teramura $M$, Kimura $A$, Iwase $S$, et al Treatment of severe aplastic anemia with antithymocyte globulin and cyclosporin A with or without G-CSF in adults: a multicenter randomized study in Japan. Blood. 2007:110(6):1756-1761.

36. Schanz J, Cevik N, Fonatsch C, et al Detailed analysis of clonal evolution and cytogenetic evolution patterns in patients with myelodysplastic syndromes (MDS) and related myeloid disorders. Blood Cancer J. 2018;8(3):28. 